Therefore, from Eq. (A3) we have

$$
P=\frac{1}{3} m C A^{2}\left|\int \Psi_{\mathrm{n}_{t}}^{*} \nabla \Psi_{\text {in }} d \tau\right|^{2}
$$

which is independent of the direction of $\mathbf{A}$.

Equation (A3) holds generally. Thus, (provided only $\Psi_{\text {in }}$ has spherical symmetry and $e^{2 \pi i \Delta \mathbf{r} \cdot \mathbf{k}}$ can be replaced by unity) the absorption coefficient $\mu(\lambda)$ is independent on the direction of $\mathbf{A}$ not only for cubic crystals but whenever the term in the square brackets in (A3) is independent of the direction of $\mathbf{A}$. It should be noted that for the purpose of this calculation the space group and not the point group is of importance, because here an $n$-fold screw axis is equivalent to an $n$-fold rotation axis and a glide plane is equivalent to a plane of symmetry, considering the summation over-all equivalent atoms.

Thus, $\mu(\lambda)$ will be the same for all those directions of A which give equal values of the expression in the square brackets of Eq. (A3). For example, from Eq. (A3) follows that when the investigated crystal possesses only a three-fold (or a higher) symmetry axis and the beam is parallel to this axis, then $\mu(\lambda)$ is independent of the direction of $\mathbf{A}$.

\title{
Electronic Structure of Copper Impurities in $\mathrm{ZnO}$
}

\author{
R. E. Dietz, H. Kamimura,* M. D. Sturge, and A. Yariv \\ Bell Telephone Laboratories, Murray Hill, New Jersey
}

(Received 6 June 1963)

\begin{abstract}
We have measured the near infrared absorption, Zeeman effect, and electron spin resonance of $\mathrm{Cu}^{2+}$ ions introduced as a substitutional impurity into single-crystal $\mathrm{ZnO}$. From the $g$ values of the lowest $\Gamma_{6}$ component of the $T_{2}$ state (the ground state), $g_{I I}=0.74$ and $g_{\perp}=1.531$, and from the $g$ values of the $\Gamma_{4} \Gamma_{5}$ component of the $E$ state, $g_{I I}=1.63$ and $g_{\perp}=0$, we have determined the wave functions of $\mathrm{Cu}^{2+}$ in terms of an LCAO MO model in which overlap only with the first nearest neighbor oxygen ions is considered. These wave functions indicate that the copper $3 d\left(t_{2}\right)$ hole spends about $40 \%$ of its time in the oxygen orbitals, and that the copper $t_{2}$ orbitals are expanded radially with respect to the $e$ orbitals. Corroboration for the radial expansion of the $t_{2}$ orbitals is obtained from an analysis of the hyperfine splitting. It is concluded from our model that the large values of the hyperfine constants, $|A|=195 \times 10^{-4} \mathrm{~cm}^{-1}$ and $|B|=231$ $\times 10^{-4} \mathrm{~cm}^{-1}$, are due to the contribution from the orbital motion of the $t_{2}$ hole.
\end{abstract}

\section{INTRODUCTION}

A LTHOUGH much work has been done on the spin resonance and optical spectra of transition metal impurities in compound semiconductors, ${ }^{1-4}$ the nature of the states remains obscure. The energy levels of these impurities are deep, so the hydrogenic model based on the effective mass formalism is not applicable. ${ }^{5}$ An alternative point of view exists, that of the ligand field theory, which was originally developed for transition ions in ionic lattices. ${ }^{6}$ The formalism of ligand field theory is based primarily on symmetry considerations, though its usefulness depends on the degree of localization of the center. The transition from ionic to covalent

* On leave from the Department of Physics, University of Tokyo, Tokyo, Japan.

1 G. W. Ludwig and H. H. Woodbury, in Solid State Physics, edited by F. Seitz and D. Turnbull (Academic Press Inc., New York, 1962), Vol. 13, 223.

2 R. Pappalardo, J. Mol. Spectry. 6, 554 (1961).

${ }^{3}$ R. Pappalardo and R. E. Dietz, Phys. Rev. 123, 1188 (1961).

${ }^{4}$ H. A. Weakliem, J. Chem. Phys. 36, 2117 (1962).

-5 W. Kohn, in Solid State Physics, edited by F. Seitz and D. Turnbull (Academic Press Inc., New York, 1957), Vol. 5, 257.

${ }^{6}$ For example, see J. S. Griffith, The Theory of TransitionMetal Ions (Cambridge University Press, Cambridge, England, 1961). bonding is taken into account by allowing the parameters used in the theory to vary. The calculation of these parameters is the task of a microscopic theory, and to our knowledge no case has so far been reported of their successful calculation for the case of strong covalent bonding such as occurs in a semiconductor..$^{7,8}$

In this paper we report a detailed investigation of the optical spectrum, Zeeman effect, and spin resonance of copper in hexagonal zinc oxide. ${ }^{9,10}$ It appears that copper substitutes for zinc, entering a slightly distorted tetrahedral site. When the Fermi level is low enough, the copper is divalent, having a single $d$ hole which in its ground state is in a triply degenerate $t_{2}$ orbital. Such an orbital is of the correct symmetry to make $\sigma$ bonds with the coordinating oxygen ions. We interpret our results in terms of a tight-binding LCAO (linear combination of

${ }^{7}$ F. S. Ham (private communication) is also investigating interstitial impurities in Si using a tight-binding model.

8 J. L. Birman, Phys. Rev. 121, 144 (1961) has also proposed a tight-binding model to explain the $\mathrm{Cu}$ centers in $\mathrm{ZnS}$. However, no calculations were attempted.

${ }^{\circ}$ H. Kamimura and A. Yariv, Bull. Am. Phys. Soc. 8, 23 (1963). M. de Wit and T. L. Estle recently reported measurements of ESR of $\mathrm{Cu}^{2+}$ in $\mathrm{ZnO}$ [Bull. Am. Phys. Soc. 8, 24 (1963)].

${ }^{10}$ R. E. Dietz, H. Kamimura, and M. D. Sturge, Bull. Am. Phys. Soc. 8, 215 (1963). 
atomic orbitals) model in which orbitals of the $\mathrm{Cu}^{2+}$ ion and of the four nearest neighbor oxygens only are included.

In Sec. II we present the near-infrared spectra and Zeeman effect of copper in zinc oxide, and for comparison, the spectra of copper in two other tetrahedral environments. These results all show the order of levels and optical selection rules predicted by ligand field theory. In Sec. III we give the spin resonance data. In Sec. IV we derive effective Hamiltonians for the ground and excited multiplets and fit them to the experimental data. A set of semiempirical parameters is thus obtained. In Sec. $\mathrm{V}$ we propose a tight-binding model to explain the small values of these parameters in the ground state, and calculate the orbital reduction factors. In order to explain the results, the model requires a radial expansion of the $d$ wave function in the ground state and about $40 \%$ delocalization of the $d$ hole. In Sec. VI we calculate the hyperfine interaction with the copper nucleus from our model in good agreement with experiment. In Sec. VII we discuss the assumptions and approximations made.

\section{OPTICAL SPECTRA}

Single crystals of $\mathrm{ZnO}$ containing about $0.05 \% \mathrm{CuO}$ were grown from a $\mathrm{PbO}$ flux by slow cooling. Most of the crystals were in the form of flat plates, the plane of which was normal to the $c$ axis. A few crystals, however, grew in the form of hollow hexagonal prisms, from which slabs containing the $c$ axis of the $\mathrm{ZnO}$ were obtained.

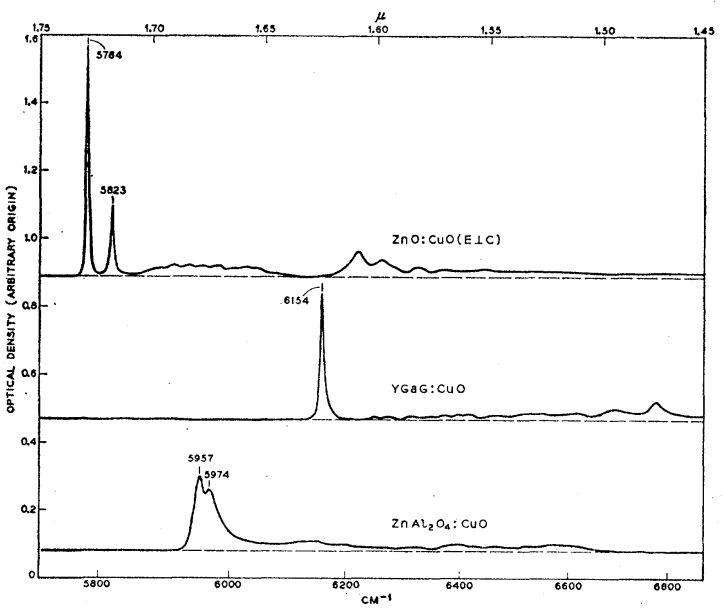

FIg. 1. Near-infrared absorption of copper in substitutiona tetrahedral sites in three oxide crystals at $4.2^{\circ} \mathrm{K}$. The (a) spectrum is that of copper in the trigonally distorted zinc site of $\mathrm{ZnO}$, with the electric vector of the light polarized normal to the $c$ axis $(E \perp c)$. Lines 5784 and 5832 are transitions $a$ and $b$, respectively, of Fig. 2. The weaker lines lying at higher energies than these sharp no-phonon lines involve excited vibrational states or the emission of phonons. Spectrum (b) is that of copper in the tetragonally distorted gallium sites of yttrium gallium garnet. Another electronic line (not shown here, but see the reference by Pappalardo) with a similar phonon train is observed at about 7334 $\mathrm{cm}^{-1}$. Finally, the spectrum of copper in the cubic tetrahedral site of zinc in $\mathrm{ZnAl}_{2} \mathrm{O}_{4}$ is given in (c).
Optical spectra were recorded using a Cary Model $14 \mathrm{R}$ Spectrophotometer or a $1.8 \mathrm{~m}$ Jarrell-Ash scanning spectrometer using a cooled $\mathrm{PbS}$ detector. Spectra were recorded between about $3 \mu$ and the band gap of the $\mathrm{ZnO}$. Apart from a variable absorption tail extending down from the intrinsic $\mathrm{ZnO}$ edge, the low-temperature spectrum consists of two sharp electronic lines at 5784 and $5823 \mathrm{~cm}^{-1}$ followed by a series of vibrational bands extending to higher frequencies. This is shown for $\sigma$ polarization (electric vector of the light plane polarized perpendicular to the $c$ axis) in Fig. 1 (a)..$^{11}$ This spectrum is observed for propagation vectors both along and normal to the $c$ axis, indicating the transition to be of the electric dipole type. In Fig. 1 (b) and 1 (c) are shown the spectra of two other oxide crystals containing copper in tetrahedral sites for comparison. As discussed

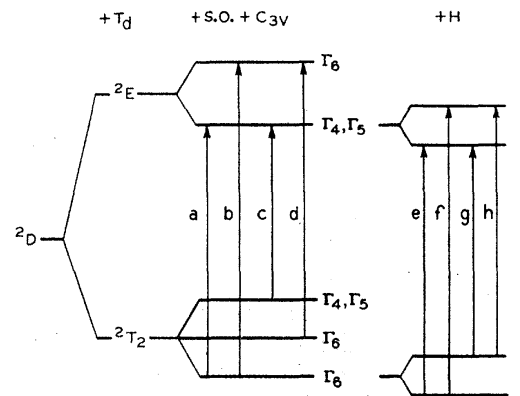

FIG. 2. Energy level diagram of the $\mathrm{Cu}^{2+}$ ion showing the progressive reduction of degeneracy of the free-ion term ${ }^{2} D$ by the application of fields of lower symmetry. The diagram is drawn for the case of a $C_{3 v}$ crystal field, corresponding to a trigonal compression along one of the bonding axes. The $E$ state is split in first order by a tetragonal distortion, but is split only in second order by an interplay of spin-orbit and trigonal fields. Thus, the combined action of these fields split the free-ion ${ }^{2} D$ state into five Kramers' doublets, which are indexed here according to the $C_{3 v}$ double group representations. With the exception of $c$, the transitions indicated by the arrows have been observed. The fact that the 5784 line is completely polarized for $E \perp c$, while the 5823 line is seen also for $E \| c$ determines that the ground state has the symmetry $\Gamma_{6}$, and that the 5784 transition terminates on the $\Gamma_{4}$, $\Gamma_{5}$ component of the ${ }^{2} E$ state.

in Refs. 2, 3, and 4, and in Sec. IV of this paper, the polarizations and splittings of the lines in these crystals are consistent with the transitions of a $d$ hole in the appropriately distorted tetrahedral coordination. Thus, it seems reasonable to adopt a tight-binding model in which a significant part of the copper wave function consists of atomic copper $3 d$ orbitals.

The states of a $d$ hole in a trigonally distorted tetrahedral field are shown in Fig. 2. The free-ion term ${ }^{2} D$ of the $\mathrm{Cu}^{2+}$ ion is split by the cubic tetrahedral field into the ${ }^{2} E$ and ${ }^{2} T_{2}$ states. The remaining orbital degeneracy

11 The spectra of copper-doped $\mathrm{ZnO}$ and yttrium gallium garnet have been reported previously by $\mathrm{R}$. Pappalardo, J. Mol. Spectry 6, 554 (1961), while the spectra of copper-doped $\mathrm{ZnO}$ have been reported by $R$. Pappalardo and R. E. Dietz, Phys. Rev. 123, 1188 (1961) and by H. A. Weakliem, J. Chem. Phys. 36, 2117 (1962). The assignments for transitions $a$ and $b$ are identical to those of Weakliem. 
of these levels is lifted by a combination of spin-orbit coupling and the trigonal field, forming five Kramer's doublets. Lines 5784 and 5823 of Fig. 1 (a) are assigned, respectively, to transitions $a$ and $b$. At $78^{\circ} \mathrm{K}$, a weak absorption at $5707 \mathrm{~cm}^{-1}$ is observed at lower frequencies than the lowest lying electronic line, as shown in Fig. 3 for $E \| c$. This absorption is believed to arise from the first thermally populated excited level $\left(\Gamma_{6}\right)$ of the $T_{2}$ multiplet (transition $d$ of Fig. 2) rather than a phonon-assisted transition from the lowest electronic level $\left(\Gamma_{6}\right)$. This assignment is not indisputable, but is probably correct in view of the following argument:

1. The strength of the 5707 line is close to the value predicted for transition $d$ by the Boltzmann factor and the theoretical ratio of oscillator strengths of $f(d) /$ $f(b)=0.8$.

2. If the 5707 line were a phonon-assisted transition of type $b$, there would be absorption on the highfrequency side of the 5823 line roughly three times as strong as is observed. (This figure is strictly correct

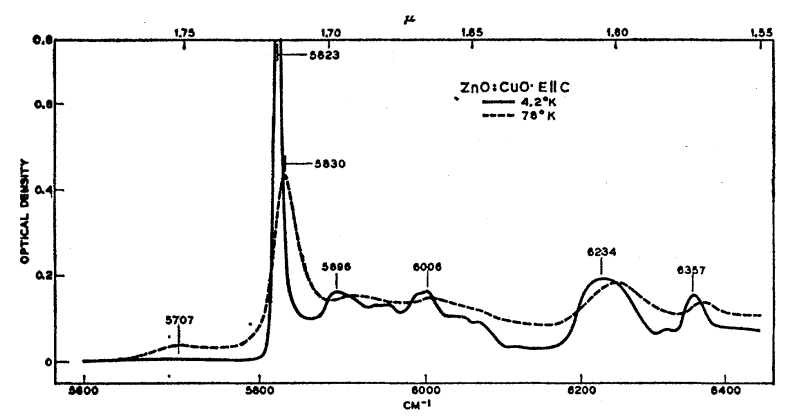

FIG. 3. The spectrum of copper-doped $\mathrm{ZnO}$ for $E \| c$. At $78^{\circ} \mathrm{K}$ an additional peak is observed at $5707 \mathrm{~cm}^{-1}$. Arguments for assigning this peak to transition $d$ of Fig. 2 are given in the text.

only in the weak-coupling limit; in the case of strongcoupling, selection rules for phonon emission and absorption could in principle be different, but, in this case, the observed simple agreement of the intensity with the Boltzmann factor would have to be fortuitous.)

At $4.2^{\circ} \mathrm{K}$, the line corresponding to transition $a$ is observed to be very narrow and to have two components split by about $0.9 \mathrm{~cm}^{-1}$, as shown in Fig. 4 (a). The ratio of the intensities of the two components is roughly that of the abundances of the two naturally occurring isotopes of $\mathrm{Cu}$. The interpretation of the apparent splitting as an isotope effect is confirmed by the observation that crystals prepared with $99.97 \%{ }^{63} \mathrm{Cu}$ show only the stronger of the two components [Fig. 4 (b)]. The longitudinal Zeeman effect of this line was measured at $23.5 \mathrm{kG}$ at $4.2^{\circ} \mathrm{K}$ and below in circularly polarized light. By observing thermalization in the ground state at $1.7^{\circ} \mathrm{K}$, we were able to distinguish between splittings of the ground and excited states. Line (b) has a limiting half-width at low temperatures of about $8 \mathrm{~cm}^{-1}$, too

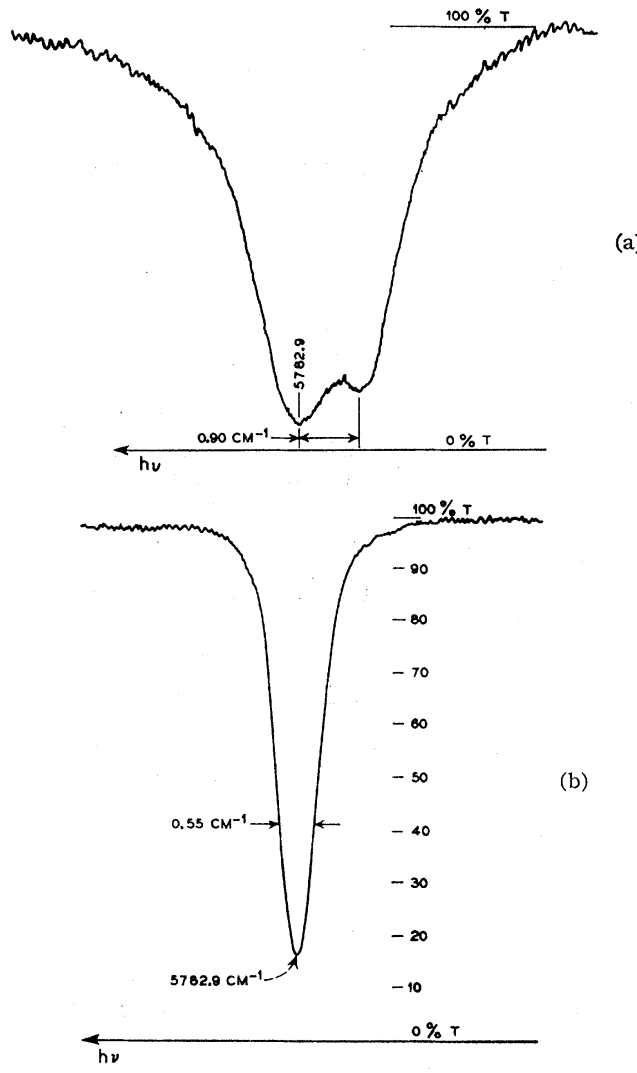

FIG. 4. (a) The 5784 line of copper-doped $\mathrm{ZnO}$ shown for $E \perp c$ at $4.2^{\circ} \mathrm{K}$ using the $1.8 \mathrm{~m}$ Ebert spectrometer. The splitting is caused by an isotope effect: the $70 \%$ abundant ${ }^{63} \mathrm{Cu}$ gives rise to the more intense line, the ${ }^{65} \mathrm{Cu}$ to the less intense line. The frequencies are corrected to vacuum for the refractive index of air. (b) The same transition in a crystal doped with $99.97 \%{ }^{63} \mathrm{Cu}$. The half-width of this line, which varies from crystal to crystal, presumably because of strain, is only $0.55 \mathrm{~cm}^{-1}$.

broad for useful Zeeman measurements. The $g$ factors obtained from these optical splittings and from electron spin resonance (ESR) measurements are given in Table I.

The positive sign of the optical $g$ factor of $\Gamma_{6}\left({ }^{2} T_{2}\right)$ for $H \| c$ was obtained from the sense of the circular polarization. ${ }^{12}$ It should be noted that the above $g$ shifts are quite large compared to those observed for $\mathrm{Cu}^{2+}$ in octahedral coordination.

The angular dependence of the Zeeman splitting is given in Fig. 5, and a tracing of the Zeeman spectrum

TABLE I. The $g$ factors for $\Gamma_{6}\left({ }^{2} T_{2}\right)$ and $\Gamma_{4} \Gamma_{5}\left({ }^{2} E\right)$ states.

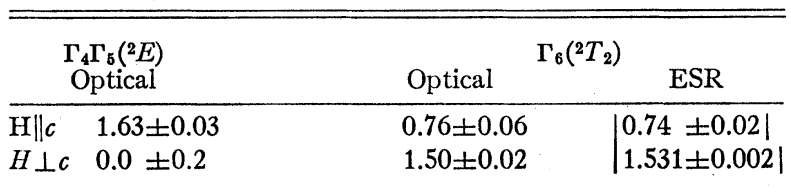

${ }^{12}$ The negative sign of this $g$ factor reported in the abstract published in Phys. Rev. Letters 11, A4 (1963). 


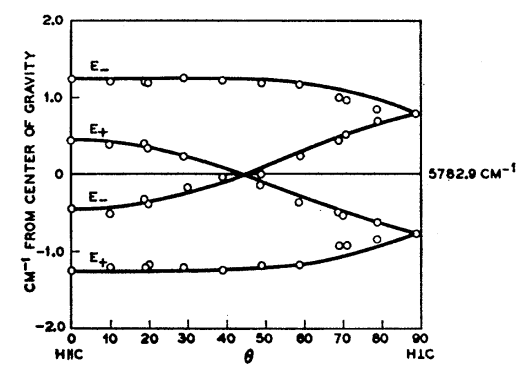

Fig. 5. Angular dependence of the Zeeman effect of the 5824 $\mathrm{cm}^{-1}$ absorption line of copper-doped $\mathrm{ZnO}$. The points are positions of the observed absorption peaks at $H=23.5 \mathrm{kG}, T=4.2^{\circ} \mathrm{K}$; the lines are calculated from Eq. (3), using the $g$ values of Table I.

for the two directions of circularly polarized radiation is given in Fig. 6. The relative intensities of transitions $e: f=h: g=0.64$.

\section{SPIN RESONANCE}

The spin resonance experiments were conducted at $\sim 23 \mathrm{kMc} / \mathrm{sec}$ and at $\sim 9 \mathrm{kMc} / \mathrm{sec}$ using conventional frequency stabilized ESR spectrometers with superheterodyne detection. ${ }^{9}$ Accurate magnetic field measurements were obtained with the aid of an NMR probe and a frequency counter. The latter was also used to determine the frequency. The measurements were performed at $1.2^{\circ} \mathrm{K}$. No spin resonance could be detected at $77^{\circ} \mathrm{K}$.

The observed spectrum was fully consistent with a spin Hamiltonian

$$
\begin{aligned}
\mathscr{F}=\beta g_{I I} S_{z} H_{z}+\beta g_{\perp}\left(H_{x} S_{x}+H_{y} S_{y}\right) & \\
& +A I_{z} S_{z}+B\left(I_{x} S_{x}+I_{y} S_{y}\right),
\end{aligned}
$$

with the " $z$ " direction taken along the $c$ axis, and

$$
\begin{aligned}
I & =\frac{3}{2}, \quad S=\frac{1}{2}, \\
\left|g_{11}\right| & =0.74 \pm 0.02, \quad\left|g_{\perp}\right|=1.531 \pm 0.002, \\
|B| & =(231 \pm 4) \times 10^{-4} \mathrm{~cm}^{-1}, \\
|A| & =(195 \pm 10) \times 10^{-4} \mathrm{~cm}^{-1},
\end{aligned}
$$

and zero quadrupolar nuclear interaction. The resonance linewidth varied from $\Delta H \sim 50 \mathrm{G}$ at $\theta=90^{\circ}$ to $\Delta H \sim 300$ $\mathrm{G}$ at $\theta=0^{\circ}$. The accuracy limits of $g_{\mathrm{II}}, g_{\perp}, A$, and $B$ reported above are due to this large linewidth. In evaluating the spin Hamiltonian parameters, corrections had to be applied for the large second-order shift

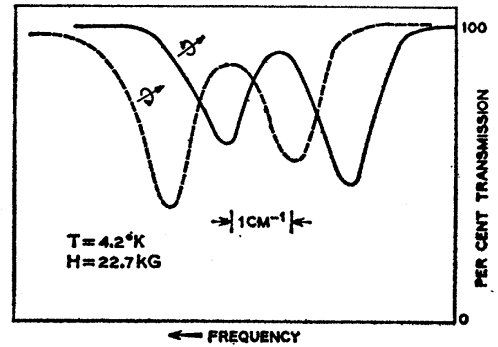

Fig. 6. Tracing of the longitudinal Zeeman spectrum for the two senses of circular polarization. in the position of the hyperfine components. The four hyperfine lines are not equally spaced but are shifted in such a way that the high-field spacing is increased by $\delta H$, the central one is unchanged, and the low-field spacing is decreased by $\delta H$, where

$$
\begin{aligned}
\delta H & =\frac{B^{2}}{2 g^{2} \beta^{2} H_{0}}\left[\frac{A^{2}+K^{2}}{K^{2}}\right], \\
g^{2} & =g_{\| 1}{ }^{2} \cos ^{2} \theta+g_{\perp}^{2} \sin ^{2} \theta, \\
K^{2} g^{2} & =A^{2} g_{11}{ }^{2} \cos ^{2} \theta+B^{2} g_{\perp}{ }^{2} \sin ^{2} \theta,
\end{aligned}
$$

and $\theta$ is the angle between the direction of the magnetic field and that of the crystal $c$ axis.

The combination of large values for $A$ and $B$ plus the small $g$ values resulted in values of $\delta H$ which were $\sim 10 \%$ of the hyperfine splittings. Another consequence of the large hyperfine interaction was a shift of $+\frac{1}{8} \delta H$ in the position of the center of the hyperfine quartet which had to be included in the determination of the $g$ values.

The separate structure of the $\mathrm{Cu}^{63}$ and $\mathrm{Cu}^{65}$ isotopes was not resolved so that the isotope shifts are smaller than the uncertainties in $g_{11}, g_{1}, A$, and $B$ as reported above.

As will be shown in Sec. VI, the anomalously large values of $A$ and $B$, compared to those observed for other transition metal ions in octahedral and tetrahedral coordination, are due to the contribution of the unquenched orbital angular momentum of the ground ${ }^{2} T_{2}$ state.

\section{SEMIEMPIRICAL ANALYSIS}

Before attempting to construct a detailed model to account for these data, we will apply to them the usual effective Hamiltonian theory. ${ }^{13}$ The effective Hamiltonian is set up so as to produce the invariant forms appropriate to the symmetry of the problem under consideration, and it does not depend on the details of bonding since arbitrary parameters are substituted for all matrix elements appearing in the expressions for the observables.

The energy levels for the ${ }^{2} E$ state of $\mathrm{Cu}^{2+}$ in a tetragonally and trigonally distorted tetrahedral site are obtained, respectively, by solving the following effective Hamiltonians :

and

$$
\mathcal{H}_{\text {eff }}(\text { tetragonal })=D V_{0}(E),
$$

$$
\mathcal{H}_{\text {eff }}(\text { trigonal })=D S_{z} T\left(A_{2}\right),
$$

where $D$ is a parameter, $z$ is a trigonal axis, and $V_{0}(E)$ and $T\left(A_{2}\right)$ are operators whose matrix representations

${ }^{13} \mathrm{Y}$. Tanabe and H. Kamimura, J. Phys. Soc. Japan 13, 394 (1958). 
are, respectively,

$$
V_{0}(E)=\left\|\begin{array}{cc}
u & v \\
& \\
& -1
\end{array}\right\|
$$

and

$$
T\left(A_{2}\right)=\left\|\begin{array}{cc}
u_{+} & u_{-} \\
& -1
\end{array}\right\| .
$$

The basic orbital functions $(u, v)$ are of the form;

$$
\begin{aligned}
u & =R_{e}(r)(\sqrt{ } 5 / 4 \sqrt{ } \pi)\left(3 Z^{2}-r^{2}\right) / r^{2}, \\
v & =R_{e}(r)(15 / 16 \pi)^{1 / 2}\left(X^{2}-Y^{2}\right) / r^{2},
\end{aligned}
$$

where $R_{e}(r)$ is a radial part of the $3 d(e)$ wave function and $(X, Y, Z)$ is a coordinate referred to the cubic axis (Fig. 7). $u_{+}$and $u_{-}$are trigonal bases which are transformed from $u$ and $v$ by

$$
\begin{aligned}
& u_{+}=-(1 / \sqrt{2})(u+i v), \\
& u_{-}=(1 / \sqrt{2})(u-i v) .
\end{aligned}
$$

As seen from the Hamiltonian, in a tetragonal distortion the ${ }^{2} E$ state is split in first order, and, thus, $D$ will directly give a measure of the splitting. On the other hand, in a trigonal distortion the ${ }^{2} E$ state is split in second order by an interplay of spin-orbit and trigonal fields, so that $D$ is given by

$$
D=4 K^{\prime} \zeta^{\prime} / \Delta E \text {. }
$$

$\Delta E$ is the splitting of the ${ }^{2} E$ and ${ }^{2} T_{2}$ states, and $K^{\prime}$ and $\zeta^{\prime}$ are defined as

$$
\begin{gathered}
K^{\prime}=-(1 / \sqrt{2})\left(t_{2} x_{+}\left|v_{\text {trig }}\right| e u_{+}\right), \\
\zeta^{\prime}=-\sqrt{2}\left(t_{2} x_{+} \frac{1}{2}\left|v_{\text {so }}\right| e u_{+} \frac{1}{2}\right) .
\end{gathered}
$$

$v_{\text {trig }}$ and $v_{\text {so }}$ are the single electron operators of the trigonal field and the spin-orbit coupling, respectively. These considerations are consistent with the large $\left(1180 \mathrm{~cm}^{-1}\right)$ splitting observed for $\mathrm{Cu}$ in the tetragonally distorted tetrahedral site of the garnet as compared with the small $\left(39 \mathrm{~cm}^{-1}\right)$ splitting of the ${ }^{2} E$ state for $\mathrm{Cu}$ in the trigonal site of $\mathrm{ZnO}{ }^{14}$ These facts indicate that it is reasonable to adopt a usual localized model for an excited $e$ hole of $\mathrm{Cu}^{2+}$ in $\mathrm{ZnO}$.

On the other hand, the effective Hamiltonian for the ${ }^{2} \mathrm{~T}_{2}$ state of $\mathrm{Cu}^{2+}$ in $\mathrm{ZnO}$ is given by

$$
\mathfrak{H}_{\text {eff }}=K V_{0}-\zeta_{\perp}\left(S_{x} T_{x}+S_{y} T_{y}\right)-\zeta_{11} S_{z} T_{z},
$$

where $3 K$ represents the energy splitting of the ${ }^{2} T_{2}$ state by the trigonal field, $\zeta_{\mathrm{II}}$ and $\zeta_{\perp}$ are the (anisotropic) spin-orbit coupling constants of the single $t_{2}$ electron,

\footnotetext{
${ }_{14}$ The tetrahedral site of the normal spinel $\mathrm{ZnAl}_{2} \mathrm{O}_{4}$ has cubic symmetry, so the $17-\mathrm{cm}^{-1}$ splitting of the ${ }^{2} E$ state of $\mathrm{Cu}^{2+}$ in this site is rather surprising. The site is presumably distorted by the presence of the $\mathrm{Cu}^{2+}$ ion, possibly due to a Jahn-Teller effect in the orbitally degenerate ground state; by the Frank-Condon principle this distortion will remain during the no-phonon transition to the excited state, which is therefore split.
}

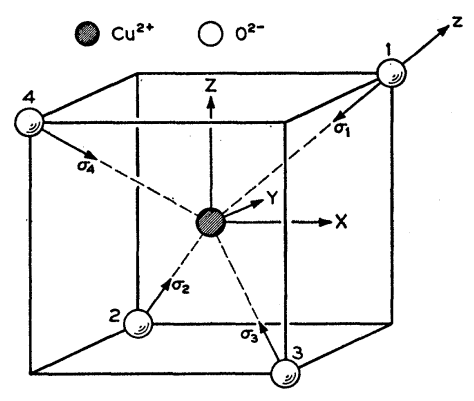

FIG. 7. The $s p^{3}$ hybridized orbitals of the four oxygens which are tetrahedrally coordinated to a central $\mathrm{Cu}$ ion. The other $s p^{3}$ hybridized orbitals of these oxygens make tetrahedral bonds with the $s p^{3}$ orbitals of the zinc ions. $(X, Y, Z)$ is a coordinate axis referred to the cube edges. $(x, y, z)$ is another cartesian system in which the $z$ axis is taken along the $c$ axis (the [111] direction).

and $V_{0}$ and $T_{x}, T_{y}$ and $T_{z}$ are the trigonal field and angular momentum operators whose matrix representations are given, respectively, by
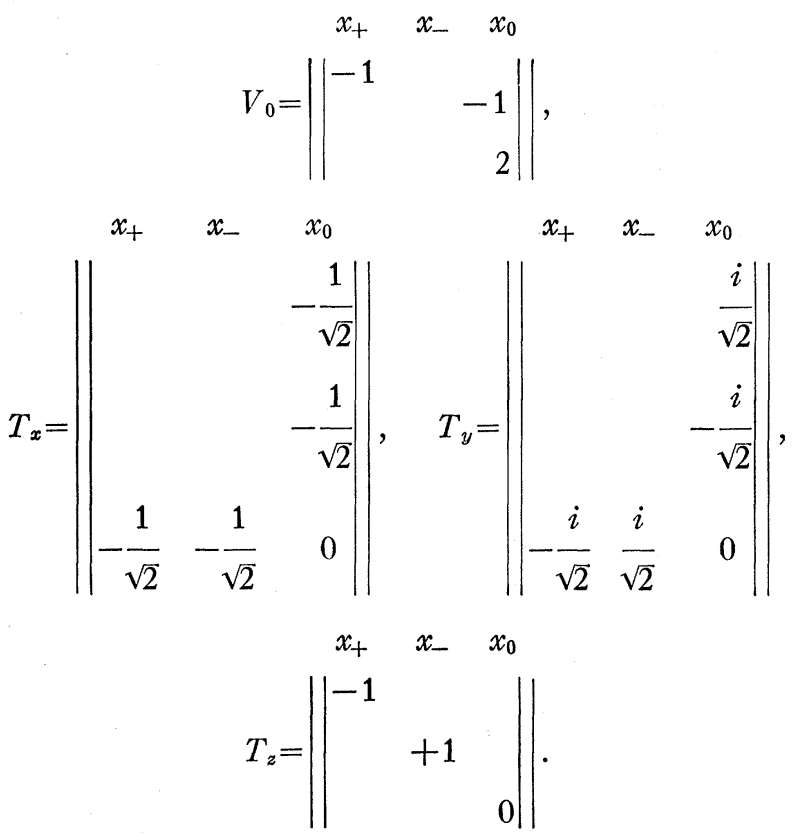

$x_{+}, x_{-}$, and $x_{0}$ are the trigonal bases of the $T_{2}$ state, corresponding to the component of the orbital angular momentum along the trigonal $(z)$ axis of $-1,+1$, and 0 , respectively.

By solving the effective Hamiltonians (6) and (13), the energy levels of the $\mathrm{Cu}^{2+}$ ion are given in the following, together with their eigenfunctions: For ${ }^{2} E$

$$
\begin{aligned}
& W_{1}=\frac{1}{\Delta E}\left(2 K^{\prime} \zeta^{\prime}-2 K^{\prime 2}-\frac{\zeta^{\prime 2}}{4}\right), \Gamma_{4}, \Gamma_{5}\left\{\begin{array}{l}
\psi\left(\frac{1}{2} u_{+}\right) \\
\psi\left(-\frac{1}{2} u_{-}\right)
\end{array},\right. \\
& W_{2}=\frac{1}{\Delta E}\left(-2 K^{\prime} \zeta^{\prime}-2 K^{\prime 2}-\frac{\zeta^{\prime 2}}{4}\right), \quad \Gamma_{6}\left\{\begin{array}{l}
\psi\left(\frac{1}{2} u_{-}\right) \\
\psi\left(-\frac{1}{2} u_{+}\right)
\end{array}\right.
\end{aligned}
$$


and for ${ }^{2} T_{2}$

$$
\begin{aligned}
& W_{3}=-K+\frac{\zeta_{11}}{2}, \quad \Gamma_{4}, \Gamma_{5}\left\{\begin{array}{l}
\psi\left(\frac{1}{2} x_{+}\right) \\
\psi\left(-\frac{1}{2} x_{-}\right)
\end{array},\right. \\
& W_{4}=-K-\frac{\zeta_{11}}{2}+\frac{\zeta_{1}}{\sqrt{2}} \tan \alpha, \\
& \Gamma_{6}\left\{\begin{array}{l}
\psi\left(\frac{1}{2} x_{0}\right) \sin \alpha+\psi\left(-\frac{1}{2} x_{+}\right) \cos \alpha \\
\psi\left(-\frac{1}{2} x_{0}\right) \sin \alpha+\psi\left(\frac{1}{2} x_{-}\right) \cos \alpha
\end{array},\right. \\
& W_{5}=-K-\frac{\zeta_{11}}{2}-\frac{\zeta_{1}}{\sqrt{2}} \cot \alpha, \\
& \Gamma_{6}\left\{\begin{array}{l}
\psi\left(\frac{1}{2} x_{0}\right) \cos \alpha-\psi\left(-\frac{1}{2} x_{+}\right) \sin \alpha \\
\psi\left(-\frac{1}{2} x_{0}\right) \cos \alpha-\psi\left(\frac{1}{2} x_{-}\right) \sin \alpha
\end{array},\right.
\end{aligned}
$$

where

$$
\tan 2 \alpha=-\sqrt{2} \zeta_{\perp} /\left(3 K+\frac{\zeta_{11}}{2}\right), \quad 0 \leq \alpha \leq \frac{\pi}{2}
$$

and $\pm \frac{1}{2}$ in the wave functions indicates quantization of the spin with respect to the $c$ axis. In the expressions for $W_{1}$ and $W_{2}$ second-order terms producing shifts of ${ }^{2} E$ as a whole have been included.

Note that the state $W_{5}\left(\Gamma_{6}\right)$ is the lowest energy state irrespective of the sign of the trigonal field $K$.

By using the eigenfunctions (14a) and (15c), we will derive the $g$ values for the lowest $\Gamma_{6}$ Kramers' doublet of the ${ }^{2} T_{2}$ state, and the $\left(\Gamma_{4}, \Gamma_{5}\right)$ Kramers' doublet of the ${ }^{2} E$ state which will be compared with the experimental results.

Since $\left(u_{ \pm}\left|T_{z}\right| u_{ \pm}\right)=0$ and $\left(u_{+}\left|T_{x}\right| u_{-}\right)=0$,

and

$$
g_{\text {II }}\left(\Gamma_{4}, \Gamma_{5}\left({ }^{2} E\right)\right)=2
$$

$$
g_{\perp}\left(\Gamma_{4}, \Gamma_{5}\left({ }^{2} E\right)\right)=0 .
$$

By comparing these values with experimental values of $g_{11}{ }^{\mathrm{ex}}=1.63$ and $g_{\perp}{ }^{\mathrm{ex}}=0.0 \pm 0.2$, it is clear that the configuration mixing effect of the ${ }^{2} T_{2}$ state plays an important role in producing the large $g$ shift of the ${ }^{2} E$ state. Therefore, taking the configuration mixing of the ${ }^{2} T_{2}$ state by the spin-orbit and trigonal field into account, the eigenfunctions of the $\Gamma_{4}, \Gamma_{5}\left({ }^{2} E\right)$ component

\begin{tabular}{|c|c|c|c|c|c|}
\hline \multirow{2}{*}{${ }^{2} T_{2} \gamma{ }^{2} E \gamma^{\prime}$} & \multicolumn{2}{|c|}{$\left({ }^{2} T_{2} \gamma\left|T_{z}\right|{ }^{2} E \gamma^{\prime}\right)$} & \multirow{2}{*}{${ }^{2} T_{2 \gamma}{ }^{2} E \gamma$} & \multicolumn{2}{|c|}{$\left({ }^{2} T_{2 \gamma}\left|T_{x}\right|^{2} E \gamma^{\prime}\right)$} \\
\hline & $u_{+}$ & $u_{-}$ & & $u_{+}$ & $u_{-}$ \\
\hline $\begin{array}{l}x_{+} \\
x_{-} \\
x_{0}\end{array}$ & $\begin{array}{c}-\sqrt{2} k^{\prime} \\
0 \\
0\end{array}$ & $\begin{array}{c}0 \\
\sqrt{2} k^{\prime} \\
0\end{array}$ & $\begin{array}{l}x_{+} \\
x_{-} \\
x_{0}\end{array}$ & $\begin{array}{l}0 \\
k^{\prime} \\
k^{\prime}\end{array}$ & $\begin{array}{c}-k^{\prime} \\
0 \\
k^{\prime}\end{array}$ \\
\hline
\end{tabular}
are written in the following:

$$
\begin{gathered}
\varphi_{+}=\frac{1}{\sqrt{ } L}\left\{\psi\left(\frac{1}{2} u_{+}\right)+\frac{1}{\Delta E}\left(\frac{\zeta^{\prime}}{\sqrt{2}}+\sqrt{2} K^{\prime}\right) \psi\left(\frac{1}{2} x_{+}\right)\right. \\
\left.+\frac{-\zeta^{\prime}}{\Delta E} \psi\left(-\frac{1}{2} x_{-}\right)\right\}, \\
\varphi_{-}=\frac{1}{\sqrt{ } L}\left\{\psi\left(-\frac{1}{2} u_{-}\right)+\frac{\zeta^{\prime}}{\Delta E} \psi\left(\frac{1}{2} x_{+}\right)\right. \\
\left.+\frac{1}{\Delta E}\left(\frac{\zeta^{\prime}}{\sqrt{2}}+\sqrt{2} K^{\prime}\right) \psi\left(-\frac{1}{2} x_{-}\right)\right\},
\end{gathered}
$$

where $L$ is the normalization constant.
TABLE II. The off-diagonal matrix element of $\mathbf{T}$ between the ${ }^{2} E$ and ${ }^{2} T_{2}$ states $\left({ }^{2} T_{2} \gamma|T|^{2} E \gamma^{\prime}\right)$.

By using Table II for the off-diagonal matrix elements of the orbital angular momentum $\mathbf{T}$, the $g$ values of the $\Gamma_{4}, \Gamma_{5}$ state are given by

$$
\begin{aligned}
g_{\text {II }}\left(\Gamma_{4}, \Gamma_{5}\left({ }^{2} E\right)\right)=2\left[\left(1-2 k^{\prime}\left(2 K^{\prime}\right.\right.\right. & \left.\left.+\zeta^{\prime}\right) / \Delta E\right)^{2} \\
& \left.+8\left(\zeta^{\prime} k^{\prime} / \Delta E\right)^{2}\right]^{1 / 2},
\end{aligned}
$$

and

$$
g_{\perp}\left(\Gamma_{4}, \Gamma_{5}\left({ }^{2} E\right)\right)=0,
$$

where $k^{\prime}=-(1 / \sqrt{2})\left(t_{2} x_{+}\left|l_{z}\right| e u_{+}\right) . l_{z}$ is the single-electron operator of the $z$ (trigonal) component of the orbital angular momentum.

Similarly, taking into account the configuration mixing of the ${ }^{2} E$ state and further the mixing of the $4 P$ state $^{15}$ due to the tetrahedral potential of the form $A X Y Z$, the eigenfunctions of the lowest Kramers' doublet $\Gamma_{6}\left({ }^{2} T_{2}\right)$ are expressed by

$$
\begin{aligned}
|-\rangle= & (1 / \sqrt{ } M)\left\{\left[\psi\left(\frac{1}{2} x_{0}\right)+\left(\zeta^{\prime} / \Delta E\right) \psi\left(-\frac{1}{2} u_{+}\right)\right.\right. \\
& \left.+c^{2} \varphi_{4 P}\left(\frac{1}{2} a_{0}\right)\right] \cos \alpha-\left[\psi\left(-\frac{1}{2} x_{+}\right)\right. \\
& \left.\left.+\frac{\zeta^{\prime}-2 K^{\prime}}{\sqrt{2} \Delta E} \psi\left(-\frac{1}{2} u_{+}\right)+c^{2} \varphi_{4 P}\left(-\frac{1}{2} a_{+}\right)\right] \sin \alpha\right\}
\end{aligned}
$$

and

$$
\begin{aligned}
|+\rangle=(1 / \sqrt{ } M)\left\{\left[\psi\left(-\frac{1}{2} x_{0}\right)+\left(\zeta^{\prime} / \Delta E\right) \psi\left(\frac{1}{2} u_{-}\right)\right.\right. \\
\left.+c^{2} \varphi_{4 P}\left(-\frac{1}{2} a_{0}\right)\right] \cos \alpha-\left[\psi\left(\frac{1}{2} x_{-}\right)\right. \\
\left.\left.\quad+\frac{\zeta^{\prime}-2 K^{\prime}}{\sqrt{2} \Delta E} \psi\left(\frac{1}{2} u_{-}\right)+c^{2} \varphi_{4 P}\left(\frac{1}{2} a_{-}\right)\right] \sin \alpha\right\} .
\end{aligned}
$$

Here $M$ is the normalization constant and $\varphi_{4 P}$ 's are the wave functions of the $\mathrm{Cu}^{2+} 4 P$ state with $a_{0}=(1 / \sqrt{3})$ $\times\left(P_{X}+P_{Y}+P_{Z}\right), a_{+}=-(1 / \sqrt{3})\left(\omega P_{X}+\omega^{2} P_{Y}+P_{Z}\right)$ and $a_{-}=(1 / \sqrt{3})\left(\omega^{2} P_{X}+\omega P_{Y}+P_{Z}\right)$, where $\omega=e^{2 \pi i / 3}$.

By using these eigenfunctions, the $g$ values defined in the spin Hamiltonian (1) are calculated in the following:

$$
\begin{array}{r}
g_{11}=2\left(+\left|T_{z}+2 S_{z}\right|+\right)=-2\left\{\cos 2 \alpha-k_{11} \sin ^{2} \alpha\right. \\
\left.-\frac{k^{\prime}}{\Delta E}\left(2\left[\zeta^{\prime}-2 K^{\prime}\right] \sin ^{2} \alpha-\sqrt{2} \zeta^{\prime} \sin 2 \alpha\right)\right\},
\end{array}
$$

${ }^{15}$ The $\mathrm{Cu}^{2+} 4 P$ state is mixed with crystal orbitals of the same symmetry; this does not affect the argument, here or in Sec. VI. 
and

$$
\begin{gathered}
g_{\perp}=2\left(+\left|T_{x}+2 S_{x}\right|-\right)=2\left\{\cos ^{2} \alpha+\frac{k_{\perp}}{\sqrt{2}} \sin 2 \alpha\right. \\
\left.-\frac{k^{\prime}}{\Delta E}\left[\frac{\left(\zeta^{\prime}-2 K^{\prime}\right)}{\sqrt{2}} \sin 2 \alpha-2 \zeta^{\prime} \cos ^{2} \alpha\right]\right\},
\end{gathered}
$$

where

$$
\begin{aligned}
k_{11} & =-\left(t_{2} x_{+}\left|l_{z}\right| t_{2} x_{+}\right), \\
k_{\perp} & =\left(t_{2} x_{+}\left|l_{+}\right| t_{2} x_{0}\right) .
\end{aligned}
$$

Here $l_{+}$is the single-electron operator of the $(x+i y)$ component of the orbital angular momentum. In these expressions we have dropped the term representing the mixing of the $\mathrm{Cu} 4 P$ state. From the oscillator strength of $2 \times 10^{-4}$ we can estimate an upper limit of $10^{-2}$ for the $4 P$ mixing coefficient $c^{2}$. This is too small to affect the $g$ values.

The quantities $k_{\mathrm{II}}, k_{\perp}$, and $k^{\prime}$ are the "orbital reduction factors" which are equal to unity for pure $d$-wave functions. Since we explicitly took configuration mixing into account in the expressions (19a) and (19b), deviations of $k_{11}, k_{1}$, and $k^{\prime}$ from unity can be attributed to the so-called covalency effect.

We now determine the parameters so as to fit to the experimental data. In doing so, we assume isotropy in the spin-orbit coupling constants and orbital reduction factors, that is $k_{I I}=k_{\perp}$ and $\zeta_{11}=\zeta_{\perp}$. The approximation for $k_{11}=k_{\perp}$ is justified by the smallness of the trigonal field. This is not true for $\zeta$, which if small can have appreciable anisotropy due to configuration mixing.

The excited-state parameters $k^{\prime}, \zeta^{\prime}$, and $K^{\prime}$ are determined from the excited $g_{11}$ and the splitting of the ${ }^{2} E$ state by assuming

$$
k^{\prime}=\zeta^{\prime} / \zeta_{\mathrm{FI}},
$$

where $\zeta_{\text {FI }}$ is the $\mathrm{Cu}^{2+}$ free-ion spin-orbit constant $\left(\zeta_{\mathrm{FI}}=830 \mathrm{~cm}^{-1}\right)$. This assumption is reasonable if the semiempirical values of parameters $\zeta^{\prime}$ and, therefore, $k^{\prime}$ being thus determined are not greatly different from the free-ion values. In fact, the results obtained show that this assumption is reasonable. $\alpha$, that is, $K / \zeta$, and $k$ are determined from the $g$ factors of the ground state, the configuration mixing terms being calculated from $k^{\prime}, \zeta^{\prime}$, and $K^{\prime}$. Then $\zeta$ is determined from the groundstate splitting between two $\Gamma_{6}$ states $\left(=123 \mathrm{~cm}^{-1}\right)$ using the value of $\alpha$ obtained above. Finally, $K$ is determined from the ratio $K / \zeta$ which was determined from the $g$ factors of the ground state. The semiempirical values of all parameters thus obtained are tabulated in the Table III. It is seen that the spin-orbit coupling constants and the orbital angular momentum within the $T_{2}$ manifold are small compared to their free-ion values. However, the values obtained for $K$ and $\zeta$ seem to be anomalously small, considering the value for
TABLE III. The semiempirical values of the parameters, with their definitions.

$$
\begin{aligned}
\Delta E & =10 D q=5690 \mathrm{~cm}^{-1} \\
\zeta & =-2\left(t_{2} x_{+} \frac{1}{2}\left|v_{\mathrm{so}}\right| t_{2} x_{+} \frac{1}{2}\right)=86 \mathrm{~cm}^{-1}\left(522 \mathrm{~cm}^{-1}\right) \\
\zeta^{\prime} & =-\sqrt{2}\left(t_{2} x_{+}\left|\frac{1}{2}\right| v_{\mathrm{so}} \mid e u_{+} \frac{1}{2}\right)=720 \mathrm{~cm}^{-1} \\
K & =\left(t_{2} x_{+}\left|v_{\text {trig }}\right| t_{2} x_{+}\right)=-10 \mathrm{~cm}^{-1 \mathrm{a}}\left(-57 \mathrm{~cm}^{-1}\right) \\
K^{\prime} & =-(1 / \sqrt{2})\left(t_{2} x_{+}\left|v_{\text {trig }}\right| e u_{+}\right)=-77 \mathrm{~cm}^{-1} \\
k & =-\left(t_{2} x_{+}\left|l_{z}\right| t_{2} x_{+}\right)=0.46 \\
k^{\prime} & =-(1 / \sqrt{2})\left(t_{2} x_{+}\left|l_{z}\right| e u_{+}\right)=0.87
\end{aligned}
$$

a By taking into account the anisotropy in $\zeta$ arising from the configuration mixing, the values for $K, \zeta_{11}$, and $\zeta_{1}$, are found to be $+0.4 \mathrm{~cm}^{-1}, 27 \mathrm{~cm}^{-1}$,

$k$ which was obtained from the $g$ factors of the ground state. Thus, some doubt is thrown on the assignment of the 5707 line to the upper of the two $\Gamma_{6}$ components of the ground ${ }^{2} T_{2}$ state, rather than a phonon-assisted transition from the lowest electronic level $\left(\Gamma_{6}\right)$. As will be discussed in Sec. VI, a value of $\zeta$ can be estimated from the observed hyperfine splitting and then $K$ will be estimated from the value of $K / \zeta$. These values are shown in the parentheses in Table III. The negative value of $K$ is consistent with the contraction of the $\mathrm{Zn}$ site along the $c$ axis.

\section{TIGHT-BINDING LCAO MO MODEL}

In this section we consider a model to explain the small values of the $t_{2}$ parameters semiempirically determined in the previous section. Such small values of the $t_{2}$ parameters suggest that strong covalency between the copper ion and its environment must be considered. In pure $\mathrm{ZnO}$ each zinc atom is situated at the center of a tetrahedron formed by its four nearest neighbor oxygen ions, to which it is covalently bonded. In our model we assume the bonding electrons to be in the $s p^{3}$ orbitals. We further assume that when a cupric ion substitutes for zinc, the copper $d$ electrons make $\sigma$ bonds with surrounding four oxygen ions. To do this we hybridize the $\mathrm{Cu}^{2+} t_{2}$ orbitals towards the nearest neighbor oxygen ions, thus making strong $\sigma$ bonds with the $s p^{3}$ hybridized $\sigma$ orbitals of the oxygen ions which direct towards the $\mathrm{Cu}^{2+}$ ion. The remainder of the $s p^{3}$ hybridized $\sigma$ orbitals of the oxygen ions makes $\sigma$ bonds with the $s p^{3}$ orbitals of the second nearest neighbor zinc ions. Therefore, there are no oxygen orbitals left to form $\pi$ bonds with $d$ orbitals of the copper. Assuming that the adjacent bonds are not greatly affected by the $t_{2}$ hole of the $\mathrm{Cu}^{2+}$ ion, the electronic wave function for the $\sigma$ bond between the $\mathrm{Cu}$ and oxygen ions may be constructed from suitable combinations of $\mathrm{Cu} 3 d\left(t_{2}\right)$ and $\sigma$ orbitals of four oxygen ions in the LCAO MO scheme.

In $T_{d}$ symmetry, the four $s p^{3}$ hybridized orbitals $\sigma_{1}, \sigma_{2}, \sigma_{3}, \sigma_{4}$ associated with the four surrounding oxygen ions 1, 2, 3, 4 (Fig. 7) are classified into bases of 
the irreducible representations $a_{1}$ and $t_{2}$ as follows. ${ }^{16}$

$$
\begin{array}{ll}
a_{1}: & \sigma_{1}+\sigma_{2}+\sigma_{3}+\sigma_{4} ; \\
t_{2}: & \left\{\begin{array}{l}
\chi_{\xi}=\frac{1}{2}\left[\sigma_{1}+\sigma_{3}-\sigma_{2}-\sigma_{4}\right], \\
\chi_{\eta}=\frac{1}{2}\left[\sigma_{1}+\sigma_{2}-\sigma_{3}-\sigma_{4}\right], \\
\chi_{\zeta}=\frac{1}{2}\left[\sigma_{1}+\sigma_{4}-\sigma_{2}-\sigma_{3}\right] .
\end{array}\right.
\end{array}
$$

Throughout the paper we neglect the overlap between the $s p^{3} \sigma$ orbitals of the adjacent oxygen ions. $\chi_{\xi}, \chi_{\eta}, \chi_{\zeta}$ combine with the $\mathrm{Cu} t_{2}$ wave functions to form $t_{2}$-type bonding and antibonding molecular orbitals, while the $a_{1}$ oxygen orbitals and the copper $e$ orbitals are nonbonding. We now denote the $\mathrm{Cu}^{2+}$ hybridized orbitals by $\varphi_{d \sigma}\left(x_{0}\right), \varphi_{d \sigma}\left(x_{+}\right)$, and $\varphi_{d \sigma}\left(x_{-}\right)$, corresponding to the component of the orbital angular momentum along the $c$ axis, $0,-1$, and +1 , respectively. We can, therefore, write for the molecular orbitals of $T_{2}$ symmetry:

$\psi\left(t_{2}, i\right)=(1 / \sqrt{ } N)\left[\varphi_{d \sigma}(i)-\rho \chi(i)\right]$,

$$
\left(i=x_{0}, x_{+} \text {, and } x_{-}\right) \text {, }
$$

where

$$
\begin{aligned}
& \chi\left(x_{0}\right)=(1 / \sqrt{3})\left(\chi_{\xi}+\chi_{\eta}+\chi_{\xi}\right), \\
& \chi\left(x_{+}\right)=-(1 / \sqrt{3})\left(\omega \chi_{\xi}+\omega^{2} \chi_{\eta}+\chi_{\zeta}\right), \\
& \chi\left(x_{-}\right)=(1 / \sqrt{3})\left(\omega^{2} \chi_{\xi}+\omega \chi_{\eta}+\chi_{\zeta}\right),
\end{aligned}
$$

with

$$
\omega=e^{2 \pi i / 3} .
$$

The normalization factor $N$ is given by

$$
N=1-(4 / \sqrt{3}) \rho S+\rho^{2},
$$

where $S$ is the diatomic $\sigma$-type overlap integral between the $\mathrm{Cu} \varphi_{d \sigma}$ and the $0^{2-} \sigma$ orbital.

In the above expression we have shown the antibonding molecular orbitals with positive $\rho$. As one of the authors has pointed out, ${ }^{17}$ the trigonal distortion along the $c$ axis makes $\psi\left(t_{2}, x_{0}\right)$ different from $\psi\left(t_{2}, x_{+}\right)$ and $\psi\left(t_{2}, x_{-}\right)$. However, as we made the isotropic approximation in the semiempirical analysis in the previous section, we have used a single coefficient for the $t_{2}$ molecular orbitals (21).

In the ground state, the bonding $t_{2}$, nonbonding $a_{1}$, and nonbonding $e$ orbitals are filled with electrons; the antibonding $t_{2}$ orbitals possessing five electrons or one hole. Only the energy separation of the $T_{2}$ and $E$ states is known; the relative energies of the other states are unknown, as are their positions with respect to the $\mathrm{ZnO}$ levels.

Using this model, we can derive expressions for the orbital reduction factors $k$ and $k^{\prime}$, whence we can estimate the amount of the delocalization of $d$ hole. The orbital reduction factors have already been defined as

and

$$
k=-\left(t_{2} x_{+}\left|l_{z}\right| t_{2} x_{+}\right),
$$

$$
k^{\prime}=-(1 / \sqrt{2})\left(t_{2} x_{+}\left|l_{z}\right| e u_{+}\right) .
$$

${ }^{16}$ C. A. Coulson and M. J. Kearsley, Proc. Roy. Soc. (London) A241, 433 (1957).

${ }^{17}$ H. Kamimura, Phys. Rev. 128, 1077 (1962).
We have

$$
\begin{aligned}
l_{z} \varphi_{d \sigma}\left(x_{+}\right) & =-\varphi_{d \sigma}\left(x_{+}\right) \\
\int\left(l_{z} \chi\left(x_{+}\right)\right)^{*} \varphi_{d \sigma}\left(x_{+}\right) d \tau & =\int \chi\left(x_{+}\right)^{*} l_{z} \varphi_{d \sigma}\left(x_{+}\right) d \tau \\
& =-\int \chi\left(x_{+}\right)^{*} \varphi_{d o}\left(x_{+}\right) d \tau \\
& =-(2 / \sqrt{3}) S, \\
l_{z} \psi\left(u_{+}\right) & =-\sqrt{2} \varphi_{d o}\left(x_{+}\right) R_{e}(r) / R_{t_{2}}(r),
\end{aligned}
$$

so that

$$
k=1-\frac{\rho^{2}}{1-(4 / \sqrt{3}) \rho S+\rho^{2}}
$$

and

$$
k^{\prime}=\frac{a-(2 / \sqrt{3}) \rho S^{\prime}}{\left[1-(4 / \sqrt{3}) \rho S+\rho^{2}\right]^{1 / 2}} .
$$

Here $S^{\prime}$ is an integral similar to $S$ (the angular part of $S$ and $S^{\prime}$ are the same), but it contains the radial part of the $\mathrm{Cu}^{2+} e$ wave function $R_{e}(r)$ instead of the respective $t_{2}$ part $R_{t_{2}}(r)$, and $a=\int R_{t_{2}}(r) * R_{e}(r) d \tau$. Although the radial parts of the $e$ and $t_{2}$ wave functions are equal in the free ion, they do not have to be equal in the crystal, and it will be necessary to take account of this fact.

Since $S$ and $S^{\prime}$ may differ, values for $\rho$ and $S$ can be obtained from (27) and (28) only by making some assumption concerning $S^{\prime}$. If we assume free ion Hartree-Fock wave functions for the $\mathrm{Cu}^{2+} e$ orbital ${ }^{18}$ and for the $\mathrm{O}^{2-} 2 s$ and $2 p$ orbitals ${ }^{19}$ and $\mathrm{Cu}-\mathrm{O}$ distance equal to the $\mathrm{Zn}-\mathrm{O}$ distance $(1.89 \AA)$, we find $S^{\prime}=0.082$. Substituting this value in (27) and (28), assuming $a=1$ in (28), and using the semiempirical values of $k=0.46$ and $k^{\prime}=0.87$, we find $S=0.27$ and $\rho=0.78$. This yields the covalency $\Lambda=\rho^{2} /\left(1+\rho^{2}\right)=0.38$. If $S^{\prime}$ is allowed to vary between the limits $0 \leq S^{\prime} \leq S$, then $0.41 \geq \Lambda \geq 0.21$ and $0.20 \leq S \leq 0.65$. Since values of $S=0.65$ are unreasonably large, it follows that $S^{\prime} \leq S$, and we conclude that the radial extension of the $\mathrm{Cu}^{2+} t_{2}$ wave function is greater than that of $e$. It will be shown later that the same conclusion can be derived from analysis of the hyperfine data.

So far we have assumed $a=1$ in (28), but if $R_{e} \neq R_{t_{2}}$, $a$ will be less than unity. This would reduce $\rho$ by the factor $a$, and, to some extent, increase $S$. Thus, the value for the covalency $38 \%$ calculated assuming free-ion extension for the $e$ orbital and $a=1$, is probably an upper limit.

Finally it should be remarked that $k$ in (27) approaches zero as $\rho$ becomes large. This is due to the fact that the $t_{2}$ orbitals form $\sigma$ bonds with the surrounding $\sigma$ orbitals. However, in the case of octahedral coordina-

${ }^{18}$ R. E. Watson, Technical Report No. 12, Solid State and Molecular Theory Group. MIT, 1959 (unpublished).

${ }^{19}$ R. E. Watson, Phys. Rev. 111, 1108 (1958). 
tion, in which the $t_{2}$ orbitals form $\pi$ bonds, $k$ lies between 1 and $0.5 .^{20}$

\section{THEORETICAL INTERPRETATION OF THE HYPERFINE DATA}

The hyperfine interaction between the $d$ hole and the $\mathrm{Cu}$ nuclear moment is given by ${ }^{21}$

$\xi^{\mathrm{hfs}}=\frac{2 \beta \mu}{I}\left(\frac{\mathbf{l} \cdot \mathbf{I}}{r^{3}}+\frac{3(\mathbf{S} \cdot \mathbf{r})(\mathbf{I} \cdot \mathbf{r})-r^{2} \mathbf{S} \cdot \mathbf{I}}{r^{5}}+\frac{8 \pi}{3} \mathbf{I} \cdot \mathbf{S} \delta(r)\right)$,

where $\beta$ is the Bohr magneton, $\mu$ is the magnetic moment of the $\mathrm{Cu}$ nucleus, $I$ is the $\mathrm{Cu}$ nuclear spin, and $\mathbf{S}$ and $\mathbf{I}$ are, respectively, the spin and orbital angular momentum of the $d$ hole. The first term represents the interaction of the $\mathrm{Cu}$ nuclear moment with the magnetic field due to the orbital motion of the $d$ hole. The second term represents the dipole-dipole interaction between the spin magnetic moment of the hole and that of the nucleus. The last term, containing the delta-function $\delta(\nu)$, is the Fermi contact term which arises from the polarization of the $s$ electrons of the $\mathrm{Cu}^{2+}$ core by exchange interaction with the $d$ hole. By using the lowest Kramers' doublet eigenfunctions (18a) and (18b), with molecular orbitals (21) for $\psi\left(x_{+}\right), \psi\left(x_{-}\right)$ and $\psi\left(x_{0}\right)$, we can calculate the hyperfine constants $A$ and $B$ in (1). These are defined as

$$
\begin{array}{r}
A=4 \frac{\beta \mu}{I}\langle+| \frac{l_{z}}{r^{3}}+\left(\frac{3 z^{2}-r^{2}}{r_{5}} S_{z}+\frac{3 z x}{r^{5}} S_{x}+\frac{3 z y}{r^{5}} S_{y}\right) \\
+\frac{8 \pi}{3} \delta(r) S_{z}|+\rangle,
\end{array}
$$

and

$$
\begin{array}{r}
B=4 \frac{\beta \mu}{I}\langle+| \frac{l_{x}}{r^{3}}+\left(\frac{3 x^{2}-r^{2}}{r^{5}} S_{x}+\frac{3 x y}{r^{5}} S_{y}+\frac{3 x z}{r^{5}} S_{z}\right) \\
+\frac{8 \pi}{3} \delta(r) S_{x}|-\rangle,
\end{array}
$$

with $A=B$ for cubic symmetry, where the $z$ axis is taken along the $c$ axis.

In order to calculate $A$ and $B$ we express $\varphi_{d \sigma}\left(x_{+}\right)$, $\varphi_{d \sigma}\left(x_{-}\right)$, and $\varphi_{d \sigma}\left(x_{0}\right)$ in terms of eigenfunctions of $L_{z}$ ( $L=2$ ) whose eigenvalue is $M_{L}$ as follows;

$$
\begin{aligned}
& \varphi_{d \sigma}\left(x_{+}\right)=-(1 / \sqrt{3})\left[\sqrt{2} \varphi\left(M_{L}=-2\right)-\varphi\left(M_{L}=+1\right)\right], \\
& \varphi_{d \sigma}\left(x_{-}\right)=(1 / \sqrt{3})\left[\sqrt{2} \varphi\left(M_{L}=2\right)-\varphi\left(M_{L}=-1\right)\right], \\
& \varphi_{d \sigma}\left(x_{0}\right)=\varphi\left(M_{L}=0\right) .
\end{aligned}
$$

Thus, the hyperfine constants are given by

$$
\begin{aligned}
& A=4 \frac{\beta \mu}{I} \frac{1}{M}\left[\frac{1}{N}\left\langle\frac{1}{r^{3}}\right\rangle_{t_{2}}\left(\frac{2}{7} \cos ^{2} \alpha-\frac{6}{7} \sin ^{2} \alpha-\frac{1}{7 \sqrt{2}} \sin 2 \alpha\right)+c^{2}\left\langle\frac{1}{r^{3}}\right\rangle_{4 p}\left(\frac{2}{5} \cos ^{2} \alpha+\frac{6}{5} \sin ^{2} \alpha+\frac{3 \sqrt{2}}{10} \sin 2 \alpha\right)\right. \\
& \left.+2\left\langle\frac{1}{r^{3}}\right\rangle_{t_{2} e} \frac{1}{\sqrt{ } N} \frac{1}{\Delta E}\left(\frac{13 \sqrt{2}}{14} \zeta^{\prime} \sin 2 \alpha-\frac{\sqrt{2}}{7} K^{\prime} \sin 2 \alpha-\frac{2}{7} \zeta^{\prime} \cos ^{2} \alpha-\frac{12}{7}\left(\zeta^{\prime}-2 K^{\prime}\right) \sin ^{2} \alpha\right)+\frac{\cos 2 \alpha}{3} \frac{1}{N} \chi\right], \\
& B=4 \frac{\beta \mu}{I} \frac{1}{M}\left[\frac{1}{N}\left\langle\frac{1}{r^{3}}\right\rangle_{t_{2}}\left(-\frac{1}{7} \cos ^{2} \alpha-\frac{1}{7} \sin ^{2} \alpha+\frac{15}{14 \sqrt{2}} \sin 2 \alpha\right)-c^{2}\left\langle\frac{1}{r^{3}}\right\rangle_{4 p}\left(+\frac{1}{5} \cos ^{2} \alpha+\frac{3}{5} \sin ^{2} \alpha+\frac{13}{10 \sqrt{2}} \sin 2 \alpha\right)\right. \\
& \left.+2\left\langle\frac{1}{r^{3}}\right\rangle_{t_{2} e} \frac{1}{\sqrt{ } N} \frac{1}{\Delta E}\left(\frac{43}{21} \zeta^{\prime} \cos ^{2} \alpha+\frac{86 K^{\prime}-55 \zeta^{\prime}}{42 \sqrt{2}} \sin 2 \alpha+\frac{\zeta^{\prime}-2 K^{\prime}}{6} \sin ^{2} \alpha\right)+\frac{\cos ^{2} \alpha}{3} \frac{1}{N} \chi\right] \text {. }
\end{aligned}
$$

$\chi$ is a measure of the net spin density at the $\mathrm{Cu}$ nucleus :

$$
\chi=4 \pi \sum_{i s}\left[\rho_{\uparrow}^{i s}(0)-\rho_{\downarrow}^{i s}(0)\right],
$$

where $\rho_{\uparrow}{ }^{i s}$ and $\rho_{\downarrow}{ }^{i s}$ are the densities at the $\mathrm{Cu}$ nucleus of $s$ electrons of $i$ th shell with spins up and down respectively, and

$$
\begin{aligned}
& \left\langle\frac{1}{r^{3}}\right\rangle_{t_{2}}=\int_{0}^{\infty}\left|R_{t_{2}}(r)\right|^{2} \frac{1}{r^{3}} r^{2} d r, \\
& \left\langle\frac{1}{r^{3}}\right\rangle_{4 p}=\int_{0}^{\infty}\left|R_{4 p}(r)\right|^{2} \frac{1}{r^{3}} r^{2} d r, \\
& \left\langle\frac{1}{r^{3}}\right\rangle_{t_{2} e}=\int_{0}^{\infty} R_{t_{2}} *(r) R_{e}(r) \frac{1}{r^{3}} r^{2} d r .
\end{aligned}
$$

${ }^{20}$ K. W. H. Stevens, Proc. Roy. Soc. (London) A219, 542 (1953).

${ }^{21}$ A. Abragam and M. H. L. Pryce, Proc. Roy. Soc. (London) A205, 135 (1951).
The first term in the expressions for $A$ and $B$ represents the contribution of the $t_{2}$ molecular orbital part of the Kramers' doublet eigenfunction, the second that of the $\mathrm{Cu} 4 p$ part, the third that of the $\mathrm{Cu} 3 d(e)$ part, and the last the exchange polarization effect of the $\mathrm{Cu}$ core $s$ electrons. It can be shown that the orbital contribution from the $4 p$ part of the wave function tends to cancel that of the $t_{2}$ molecular orbital, while the spin contributions add. This result is opposite to that obtained by Bates $e t$ al., for $\mathrm{Cu}^{2+}$ in a tetragonally distorted tetrahedral site. ${ }^{22}$ In any case, the contribution of the $4 p$ part is very small in the present case, as mentioned in IV and we can neglect it. In this we differ from Bates et al., who suggest that this mixing is important in explaining the absence of hyperfine structure in copper dipyrromethene.

From the observed values of the hyperfine constants

${ }^{22}$ C. A. Bates, W. S. Moore, K. J. Standly, and K. W. H. Stevens, Proc. Phys. Soc. (London) 79, 73 (1962). 
TABLE IV. The semiempirical values of $\left\langle 1 / r^{3}\right\rangle_{t_{2}}$ and $\chi$ of $\mathrm{Cu}^{2+}$ in $\mathrm{ZnO}$.

\begin{tabular}{ccccc}
\hline \hline Case & & $\left\langle 1 / r^{3}\right\rangle_{t_{2}}$ (a.u.) & $\chi$ (a.u.) & $H_{c}(\mathrm{kG})$ \\
\hline I & $A>0$ and $B>0$ & 5.81 & -3.83 & -162 \\
II & $A>0$ and $B<0$ & 10.99 & -62.5 & -2640 \\
\hline \hline
\end{tabular}

$A$ and $B$ we can estimate $\left\langle 1 / r^{3}\right\rangle_{t_{2}}$ and $\chi$. In this estimation, we use for $\alpha$ the value of $48^{\circ} 24^{\prime}$ which was determined from the analysis of the $g$ factors of the ground state. And further, as in Sec. V, we assume free-ion wave functions for the $3 d(e)$ part, whence $\left\langle 1 / r^{3}\right\rangle_{e}=8.25$ a.u. (atomic units). We also assume that, in the region near the $\mathrm{Cu}$ nucleus, the $t_{2}$ wave function $\varphi_{d \sigma}$ is reduced from the $e$ wave function $\psi_{e}$ by a constant factor $q$, that is, $\varphi_{d \sigma}(r)=(1 / q) \psi_{e}(r)$, whence $\left\langle 1 / r^{3}\right\rangle_{t_{2}}$ $=\left(1 / q^{2}\right)\left\langle 1 / r^{3}\right\rangle_{e}$. The calculated values of $\left\langle 1 / r^{3}\right\rangle_{t_{2}}$ and $\chi$ depend on the signs assumed for $A$ and $B$, as shown in Table IV.

For other combinations of sign, $\left\langle 1 / r^{3}\right\rangle_{t_{2}}$ turns out to be imaginary, so these cases are physically meaningless.

In the table we show the spin density $\chi$ and the effective field $H_{c}$ at the $\mathrm{Cu}$ nucleus. $H_{c}$ is defined as $\frac{2}{3} \mu_{B} \chi$ a.u. $=4.224 \times 10^{4} \chi$ G/a.u. Abragam, Horowitz, and Pryce have found $\chi$ for $\mathrm{Cu}^{2+}$ to be -2.9 a.u. (equivalent to $H_{c}=-122 \mathrm{kG}$ ) for a range of hydrated copper salts. ${ }^{23}$ In general, the experimental values of $\chi$ for divalent iron series ions in hydrated salts are close to -3 a.u..$^{23}$ Watson and Freeman have calculated $\chi$ using so-called unrestricted Hartree-Fock wave functions which have different radial dependencies for opposite spins, even for the same azimuthal quantum number $l .{ }^{24}$ They show that the net magnetic field at the nucleus is the sum of terms of opposite sign arising from the different $s$ shells. The $1 s$ and $2 s$ shells, which lie inside themaximum of the $3 d$ spin density, have their majority (parallel) spin electrons attracted outwards by exchange interaction with the unpaired $3 d$ spins. The minority (antiparallel) spin density, therefore, preponderates in the region near the nucleus, and the contribution of these shells to the effective field is therefore negative. The effective field due to the $3 s$ shell, on the other hand, is positive, and smaller than the $1 s$ and $2 s$ contributions. This is essentially because the $3 s$ shell lies partly inside but mainly outside the $3 d$ maximum. The effective fields due to the $1 s, 2 s$, and $3 s$ shells, therefore, tend to cancel leaving a net negative field at the nucleus, i.e., $\chi$ is negative.

From the analysis of the optical data in the previous section, we expect that the $3 d\left(t_{2}\right)$ spin density is expanded outwards. Therefore, the effective field due to the $3 s$ shell is smaller than that in $\mathrm{Cu}^{2+}$ ion in hydrated salts, and, thus, we would expect $\chi$ to be large and

\footnotetext{
${ }^{23}$ A. Abragam, J. Horowitz, and M. H. L. Pryce, Proc. Roy. Soc. (London) A230, 169 (1955).

${ }_{24}$ R. E. Watson and A. J. Freeman, Phys. Rev. 123, 2027 (1961).
}

negative. On the following grounds we can rule out the case II in Table IV. The value of $\left\langle 1 / r^{3}\right\rangle_{t_{2}}$ calculated for this case is larger than the free-ion value (8.25 a.u.) so that the $3 d\left(t_{2}\right)$ wave function must be contracted relative to the free-ion wave function. This is inconsistent with the large value of $\chi$ which in itself is unreasonably large. Hence, we conclude that the effective field is $-162 \mathrm{kG},\left\langle 1 / r^{3}\right\rangle_{t_{2}}=5.81$ a.u. and $A>0, B>0$. The effective field is about 1.3 times as large as that of $\mathrm{Cu}^{2+}$ in a hydrated salt and $\left\langle 1 / r^{3}\right\rangle_{t_{2}}$ is about $30 \%$ reduction from its free-ion value. From these results it can be also concluded that the origin of the large values of $A$ and $B$ is the contribution from the orbital motion of the $t_{2}$ hole.

We can estimate the spin-orbit coupling constants $-\zeta$ and $-\zeta^{\prime}$ of the $t_{2}$ and $e$ holes from the above value of $\left\langle 1 / r^{3}\right\rangle_{t_{2}}$. Since the oxygen orbital is $\sigma$ type, there is no contribution from the spin-orbit coupling of the oxygen ions. Therefore, for $\zeta$, we can write

$$
\zeta=\frac{1}{N} \frac{\left\langle 1 / r^{3}\right\rangle_{t_{2}}}{\left\langle 1 / r^{3}\right\rangle_{\mathrm{FI}}} \zeta_{\mathrm{FI}}
$$

if we neglect the small overlap between $\sigma$ orbitals on adjacent oxygen ions. We also neglect the off-diagonal term between $\mathrm{O}^{2-}$ and $\mathrm{Cu}^{2+}$ because only the region near the $\mathrm{Cu}$ nucleus contributes significantly to $\left\langle 1 / r^{3}\right\rangle$. With the normalization factor $N=1.12$ and $\zeta_{\mathrm{FI}}=830 \mathrm{~cm}^{-1}$ we find $-\zeta=-522 \mathrm{~cm}^{-1}$. Using the value of $K / \zeta$ $(=-0.11)$ which was obtained from the $g$ factor of the ground state, we find $K=-57 \mathrm{~cm}^{-1}$. These values are in poor agreement with the values obtained from the zero-field splitting of the two $\Gamma_{6}\left({ }^{2} T_{2}\right)$ components (Table III). Thus, our model is not consistent with the very low values for $\zeta$ and $K$. On the other hand, $\zeta^{\prime}$ is calculated by

$$
\zeta^{\prime}=\frac{1}{\sqrt{ } N} \frac{\left\langle 1 / r^{3}\right\rangle_{t_{2} e}}{\left\langle 1 / r^{3}\right\rangle_{\mathrm{FI}}} \zeta_{\mathrm{FI}}
$$

Since, in our approximation, $\left\langle 1 / r^{3}\right\rangle_{t_{2} e} /\left\langle 1 / r^{3}\right\rangle_{\mathrm{FI}}=\left(\left\langle 1 / r^{3}\right\rangle_{t_{2}} /\right.$ $\left.\times\left\langle 1 / r^{3}\right\rangle_{\mathrm{FI}}\right)^{1 / 2},-\zeta^{\prime}$ is estimated to be $-654 \mathrm{~cm}^{-1}$, in good agreement with the value of $-720 \mathrm{~cm}^{-1}$ obtained from the optical data (Table III).

\section{DISCUSSION}

Two main conclusions emerge from this work. The first is that the large $g$ shifts of $\mathrm{Cu}^{2+}$ in $\mathrm{ZnO}$ can be explained in terms of a model in which the $t_{2}$ hole spends about $60 \%$ of its time on the $\mathrm{Cu}^{2+}$ ion and the remainder on the four neighboring oxygen ions. The second conclusion is that the $\mathrm{Cu}^{2+} t_{2}$ wave function is radially expanded relative to the $d$ wave function of the free ion; the $e$ wave function is not so expanded.

The second conclusion is supported by two independent sets of data; the Zeeman effect on the one hand and the hyperfine interaction on the other. 
The large delocalization of the $t_{2}$ hole raises the question whether the wave function extends appreciably beyond the nearest neighbors of the $\mathrm{Cu}^{2+}$ ion. Our model is related to that of Slater and Pauling ${ }^{25-28}$ in which a total wave function for the whole crystal is constructed from $\sigma$ bonding $s p^{3}$ hybridized orbitals. The question can then be put in this form: Are the $\sigma$ bonds between $\mathrm{Zn}$ and $\mathrm{O}$ atoms near the $\mathrm{Cu}^{2+}$ impurity appreciably perturbed by the presence of the $t_{2}$ hole? We could use, for example, the semilocalized crystal orbitals of Morita $^{29}$ and add to them $t_{2}$ antibonding orbitals of the $\mathrm{Cu}^{2+}$ ion. This calculation is complicated and has not been completed. Our model does not allow for $\pi$ bonding, and minimizes the interaction of the copper ion with next nearest neighbors. The alternative model of Wolfsberg and Helmholtz $z^{30}$ allows $\pi$ bonding, and, because of this, the $\sigma$ overlap is less. In our model, however, we have tacitly assumed that the hybridization of the nearest neighbor oxygen ions is stabilized for $s p^{3}$ according to the bonding requirements of the rest of the crystal. Since the $s p^{3}$ geometry for the oxygens also is consonant with maximum $\sigma$-bonding overlap with the copper ion $t_{2}$ orbitals, it is reasonable to assume that $s p^{3}$ hybridization produces a minimum energy for the system.

An alternative approach which should give us some idea of the radial extension of the $t_{2}$ wave function is that of band theory. From this point of view, the hole is in a tightly bound acceptor state; the oxygen atomic orbitals are the basic wave functions of the valence band. If we assume a hydrogenic model for an acceptor, ${ }^{5}$ an effective mass of $1.8 m_{0}^{31}$ and a dielectric constant of 8.5 , we find the mean radius of an acceptor state to be $2.4 \AA$. As this is barely more than one atomic spacing and as the hydrogenic model must give an upper limit to the radial extension, our assumption that the $t_{2}$ wave function only extends over the $\mathrm{Cu}^{2+}$ ion and its nearest neighbors appears to be justified.

It remains to discuss two approximations made in the semiempirical analysis of Sec. IV. The assumption that for the ${ }^{2} E$ state $k^{\prime}=\zeta^{\prime} / \zeta_{\text {FI }}$ is justified in our model.

${ }^{25}$ L. Pauling, Proc. Natl. Acad. Sci. (U. S. A.) 14, 359 (1928). ${ }^{26}$ J. C. Slater, Phys. Rev. 37, 481 (1931).

${ }^{27}$ L. A. Schmidt, Phys. Rev. 92, 1373 (1953).

${ }^{28} \mathrm{~S}$. Asano and Y. Tomishima, J. Phys. Soc. Japan 11, 644 (1956).

${ }_{29}^{29}$ A. Morita, Progr. Theoret. Phys. (Kyoto) 19, 534 (1958); A. Morita and K. Takahashi, ibid. 19, 257 (1958).

${ }_{30}$ M. Wolfsberg and L. Helmholtz, J. Chem. Phys. 20, 837 (1952).

${ }_{31}$ R. E. Dietz, J. J. Hopfield, and D. G. Thomas, J. Appl. Phys. Suppl. 32, 2282 (1961).
From (28) we can write

$$
k^{\prime}=\left(\zeta^{\prime} / \zeta_{\mathrm{FI}}\right)\left(1-2 \rho S^{\prime} / a \sqrt{3}\right) .
$$

Using $\rho=0.78, S^{\prime}=0.082$, and $a=1$, this gives $k^{\prime}=0.9$ $\times\left(\zeta^{\prime} / \zeta_{\mathrm{FI}}\right)$. A self-consistent calculation gives $\zeta=765$ $\mathrm{cm}^{-1}, K^{\prime}=-85 \mathrm{~cm}^{-1}$, and $k^{\prime}=0.85$, very little different from the results in Table III. The other approximation that $k$ and $\zeta$ for the ${ }^{2} T_{2}$ state are isotropic is more difficult to justify. It is easy to show that a large anisotropy in $k$ would not be inconsistent with our model, as such anisotropy will make very little difference to the covalency parameters.

There is, however, one puzzling piece of experimental data; that is the small intensity ratio of the transitions $e$ and $f$ (Fig. 2) which has not been taken into account in determination of the semiempirical parameters. This ratio is given on the isotropic approximation to be $\tan ^{2} \alpha$, but $\alpha=48^{\circ} 24^{\prime}$ and the observed ratio is 0.62 . Thus, the introduction of anisotropy appears to be required. However, intensities depend on so many unknown factors, in particular the characteristics of the odd parity states from which crystal field transitions derive their intensity, that the intensity data have been ignored in this analysis. The role of the anisotropy in the intensity will be discussed in another paper.

We conclude that the assumptions on which our analysis is based are sound, though more work needs to be done on the origin of the intensities of the crystal field transitions and on the effect of the impurity on more distant neighbors. Such work should not affect our qualitative conclusions.

Note added in proof. In the present work we have determined $K$ and $K^{\prime}$ from independent experimental data. According to the definitions for $K$ and $K^{\prime}$ given in Table III, if $t_{2}$ and $e$ orbitals are constructed from $d$ orbitals and $v_{\text {trig }} \propto 3 z^{2}-r^{2}, K=-K^{\prime}$. Therefore, at first glance the same sign for $K$ and $K^{\prime}$ seems unreasonable. However, in the present case, the orbitals are not pure $d$ orbitals; but even if they were, fourth order terms can change the relative sign [see, for instance, D. S. McClure, J. Chem. Phys. 36, 2757 (1962)].

\section{ACKNOWLEDGMENTS}

We are grateful to S. Geschwind, F. S. Ham, C. Herring, R. G. Shulman, R. E. Watson, G. K. Wertheim, and Y. Yafet for helpful discussions; to W. E. Blumberg for help with the spin resonance measurements; to J. A. Ditzenberger and F. H. Doleiden for technical assistance, and P. E. Freeland and E. F. Dearborn for help with the crystal growing. 